\title{
A carne consumida em São Paulo: da recomendação ao impacto ambiental
}

\author{
Aline Martins de Carvalho ${ }^{1}$, Nicole Samanta Dantas Reis ${ }^{1}$, Regina Mara Fisberg, \\ Dirce Maria Lobo Marchioni ${ }^{2}$
}

O objetivo do estudo foi analisar o impacto ambiental gerado pelo consumo de carne bovina no Município de São Paulo em 2008 e verificar sua possível redução a partir da adesão às recomendações nutricionais. Utilizaramse os dados do Inquérito de Saúde de São Paulo, composto por 1.102 adultos e idosos. Para estimativa da ingestão habitual de carne bovina, utilizaram-se dois Recordatórios Alimentares de 24 horas e o Multiple Source Method. O impacto ambiental foi estimado, considerando-se que são emitidos para produção de um quilo de carne bovina $44 \mathrm{~kg}$ de equivalentes de $\mathrm{CO}_{2}$. Observou-se que o paulistano consome mais carne que $\mathrm{O}$ recomendado, e a carne bovina é a mais consumida. Estimou-se que foram liberados 3,2 quilos de equivalentes de $\mathrm{CO}_{2} /$ por dia/pessoa, isto é, 13 milhões de toneladas durante o ano de 2008. Se o paulistano consumisse a quantidade de carne recomendada pelo Ministério da Saúde, a emissão diária seria de 1,7 quilos de equivalentes de $\mathrm{CO}_{2} /$ por dia/pessoa, quase metade da emissão atual. Conclui-se que houve um alto consumo de carne bovina, assim como alta emissão de gases de efeito estufa. A redução de consumo poderia restringir pela metade os gases emitidos, além de diminuir o risco para doenças crônicas.

Palavras-chave: carne, consumo de alimentos, impacto ambiental, recomendação.

\section{Meat intake in São Paulo: from the recommendation to the environmental impact}

The present study objective was to analyze the environmental impact caused by meat consumption in city of Sao Paulo in 2008 and to investigate its possible reduction from following nutritional recommendations. Data from Health Survey of Sao Paulo was applied, comprising 1.102 adults and elderly people. It was used two 24-hour Dietary Recalls and Multiple Source Method to estimate usual meat intake. Environmental impact was estimated considering that, to produce 1 kilogram of beef, are emitted 44 kilograms of $\mathrm{CO}_{2}$ equivalents. It was observed that population of Sao Paulo consumes more meat than the recommended, and beef is the most meat consumed. It was estimated that 3,2 kilos equivalents $\mathrm{CO}_{2} /$ day/person, i.e. 13 million tons of $\mathrm{CO}_{2}$ equivalents, were released during the 2008. If Sao Paulo's population consumed the amount of meat recommended by Brazilian Ministry of Health, daily emission would be 1,7 kilos of equivalents $\mathrm{CO}_{2} /$ day/person, almost half of current emissions. It was concluded that there was a high consumption of beef, as well as high greenhouse gases emissions. Decreasing of meat consumption could restrict emitted gases by half and also reduce risk of chronic diseases.

Key-words: meat, food consumption, environmental impact, recommendation.

\footnotetext{
${ }^{1}$ Mestre em Nutrição em Saúde Pública. Departamento de Nutrição da Faculdade de Saúde Pública da Universidade de São Paulo (USP).

${ }^{2}$ Professor Associado do Departamento de Nutrição da Faculdade de Saúde Pública da USP. Correspondência: Departamento de Nutrição, Faculdade de Saúde Pública, USP. Av. Dr. Arnaldo, 715 - Cerqueira César, São Paulo, SP. CEP 00000-000. Tel : 3061-7856, fax: 3061-7804. E-mail: marchioni@usp.br.
} 


\section{INTRODUÇÃO}

A carne bovina pode ser considerada um bom alimento para nutrição humana, pois contêm proteína de alto valor biológico, minerais e vitaminas [1]. Em 100 gramas de carne de boi são fornecidos cerca de 27 gramas de proteína, $2,7 \mathrm{mg}$ de ferro e $8,1 \mathrm{mg}$ de zinco [2].

O Ministério da Saúde brasileiro orienta a ingestão de uma porção de carne por dia, correspondente a $190 \mathrm{kcal}$, como parte de uma dieta saudável, podendo ser carne bovina, suína, de porco ou aves [1].

O World Cancer Research Fund, recomenda o consumo máximo de $500 \mathrm{~g} / \mathrm{semana}$ de carne vermelha e processada para prevenção do câncer [3]. Contudo, diversos países desenvolvidos apresentam consumo

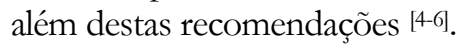

Além do impacto na saúde, outro aspecto relacionado ao consumo de carne que tem suscitado crescente interesse é sua produção, devido ao impacto ambiental gerado a partir do desmatamento, efeito estufa, poluição das águas, redução da biodiversidade e grande uso da terra para pastagem [7-11].

O presente estudo teve como objetivo analisar o impacto ambiental gerado pelo consumo de carne bovina no Município de São Paulo em 2008 e verificar sua possível redução a partir da adesão às recomendações nutricionais.

\section{METODOLOGIA}

\section{Coleta de dados e população de estudo}

Os dados do presente estudo foram obtidos a partir da pesquisa Inquérito de Saúde de São Paulo (ISA), um estudo transversal de base populacional com amostra probabilística, realizado no município de São Paulo em 2008 (ISA - Capital 2008). O processo de amostragem teve oito domínios amostrais (menores de 1 ano; crianças (1 a 11 anos); adolescentes (12 a 19 anos) de ambos os sexos; adultos (20 a 59 anos) de ambos os sexos e idosos (60 anos e mais) de ambos os sexos). O sorteio da amostra foi feito por conglomerados em dois estágios: setores censitários e domicílios. Para o presente estudo, utilizaram-se apenas os dados de indivíduos de 19 anos ou mais que responderam ao inquérito dietético, totalizando 1.102 indivíduos (585 adultos e 517 idosos). Mais detalhes da amostragem podem ser encontrados em outras publicações ${ }^{[12,13]}$.

O estudo foi submetido e aprovado pelo Comitê de Ética em Pesquisa da Faculdade de Saúde Pública da Universidade de São Paulo. Todos os participantes assinaram o Termo de Consentimento Livre e Esclarecido.

\section{Análise e processamento de dados do consumo alimentar}

Para a avaliação do consumo alimentar foram coletados dois Recordatórios Alimentares de 24 horas (R24h), sendo o primeiro R24h aplicado no domicilio usando o Multiple Pass [14] e o segundo por telefone usando o Automated Multiple Pass [15] com intervalo médio de oito meses entre eles.

O segundo R24h foi utilizado para remover a variabilidade intrapessoal, que tem efeito de inflar a distribuição, distorcendo as medidas percentilares e atenuando as medidas de efeito [16]. Para tal foi utilizada a plataforma on line Multiple Source Method (MSM), uma técnica estatística, desenvolvida para estimar a ingestão habitual de alimentos e nutrientes ${ }^{[17,18]}$.

A padronização na coleta de dados foi realizada por meio de treinamento dos entrevistadores, utilização de formulário padrão para aplicação do R24h e manual explicativo para seu preenchimento. As entrevistas ocorreram em todos os dias da semana e meses do ano.

Os dados foram criticamente revisados para padronizar e estimar a quantidade e tipo de alimentos e preparações relatados, utilizando as publicações de Pinheiro et al. [19] e Fisberg \& Villar [20]. As receitas foram desmembradas em seus constituintes, de modo a permitir a estimativa da quantidade de carne bovina presente nas preparações.

Os dados obtidos a partir do R24h foram convertidos em energia pelo software Nutrition Data System for Research [21].

\section{Impacto ambiental}

Para avaliar o impacto ambiental foi estimada a emissão de gases de efeito estufa proveniente do consumo de carne bovina em São Paulo durante o ano 
de 2008, utilizando o consumo de carne bovina como indicador de sua produção.

O consumo de carne foi estimado a partir dos dados de consumo alimentar do presente estudo. Considerou-se que a população de São Paulo no ano de 2008 era de 10.990.249 pessoas [22].

A emissão de gases de efeito estufa pela produção dessa quantidade de carne foi estimada, utilizando-se a informação que se emite cerca $44 \mathrm{~kg}$ de equivalentes de $\mathrm{CO}_{2}$ para produção de um quilo de carne bovina, o que equivale a um carro percorrendo a distância de $250 \mathrm{~km}{ }^{[11]}$.

Utilizou-se a recomendação de consumo de carne do Ministério da Saúde (190 kcal/dia) como referência para estimar a possível redução do impacto ambiental a partir da diminuição do consumo [1].

\section{Análises estatísticas}

Os valores médios e erros padrão foram calculados considerando-se a ingestão usual predita pelo Multiple Source Method [17,18]. Utilizaram-se as ponderações de amostras complexas e as análises foram estratificadas segundo sexo e faixa etária. As diferenças entre as médias foram realizadas por meio do Teste de Wald, que calcula estimativas pontuais por meio da estatística $f$, usando as ponderações de amostras complexas. Utilizou-se o software Stata 10 [23] e o valor de $p<0,05$ foi adotado como significativo.

\section{RESULTADOS}

O consumo de carne bovina e carne total da dieta, segundo sexo e faixa etária, está descrito na Tabela. Observou-se que o paulistano consome quase o dobro de carne que o recomendado pelo Ministério da Saúde e que a proporção de carne bovina em relação à carne total na dieta do paulistano é de $46,8 \%$, isto é, quase metade da carne consumida pelo paulistano é bovina.

Estimou-se que para produzir a quantidade de carne que o paulistano consome diariamente $(73,4 \mathrm{~g})$, são emitidos 3,2 quilos de equivalentes de $\mathrm{CO}_{2} /$ por dia/pessoa, isto é, 35 mil toneladas de gases de efeito estufa para a população da cidade (10.990.249 pessoas) diariamente ou 13 milhões de toneladas durante o ano. Comparativamente, cada pessoa em média produz mensalmente a mesma poluição de um carro percorrendo a distância de $550 \mathrm{~km}$.

Se o paulistano consumisse a quantidade de carne total recomendada pelo Ministério da Saúde (190 $\mathrm{kcal} / \mathrm{dia}$ ), proporcionalmente a ingestão de carne bovina seria em média $89 \mathrm{kcal} /$ dia ou cerca $40 \mathrm{~g} / \mathrm{dia}$ e a emissão diária seria de 1,7 quilos de equivalentes de $\mathrm{CO}_{2} /$ pessoa, quase metade da emissão atual.

Considerando toda a população, a redução poderia chegar a 17 mil toneladas de gases/dia, o que significa seis milhões de toneladas/ano, somente na cidade de São Paulo.

Tabela. Consumo de carne total e de boi da dieta (kcal/dia e g/dia) segundo faixa etária e sexo. São Paulo, 2008

\begin{tabular}{l|c|c|c|c|c}
\hline \multirow{2}{*}{ Carnes } & \multicolumn{2}{c|}{ Sexo } & \multicolumn{2}{c|}{ Faixa etária } & \multirow{2}{*}{ Total ** } \\
\cline { 2 - 5 } & Homem & Mulher & Adulto & Idoso & 366,7 \\
\hline Carne (todos os tipos) - kcal/dia & 466,0 & $281,1 *$ & 387,0 & $262,2 *$ & 161,8 \\
Carne (todos os tipos) - g/dia & 203,3 & $126,0 *$ & 170,3 & $117,5 *$ & 171,7 \\
Carne bovina - $\mathrm{kcal} / \mathrm{dia}$ & 230,8 & $120,7 *$ & 180,4 & $126,7 *$ & 73,4 \\
Carne bovina $-\mathrm{g} / \mathrm{dia}$ & 98,6 & $51,5 *$ & 76,9 & $55,2 *$ & 7 \\
\hline
\end{tabular}

* diferença estatisticamente significativa entre homens e mulheres e entre adultos e idosos $(p<0,05)$.

** total de carne consumida independente do sexo e faixa etária. 


\section{DISCUSSÃO}

Verificou-se grande impacto ambiental a partir do consumo de apenas um item alimentar na cidade de São Paulo. Em 2003, estimou-se que a cidade tenha consumido cerca de 410 mil toneladas de carne vermelha e processada e tenha emitido mais de 18 milhões de toneladas de equivalentes de $\mathrm{CO}_{2}$ na atmosfera [$\left[{ }^{6}\right.$. No presente estudo observou-se emissão um pouco menor, pois se considerou apenas carne bovina no estudo atual.

Observou-se também que a possível redução no consumo de carne bovina por parte da população poderia ter um grande impacto na redução das emissões de gases de efeito estufa para o meio ambiente. Entretanto, sabe-se que mudanças de hábitos alimentares são complexas, pois envolvem vários fatores como aspectos culturais, ambientais e econômicos.

Outros estudos também mostraram que a redução no consumo de carne bovina pode ajudar na redução do impacto ambiental, principalmente na redução de gases de efeito estufa [24, 25]. McMichael et al. propõe o consumo de $90 \mathrm{~g} /$ dia de carne, sendo no máximo $50 \mathrm{~g}$ de carne de ruminantes ${ }^{[25]}$.

Além do impacto ambiental dos gases de efeito estufa, desmatamento para obter pastagens, da poluição das águas e redução da biodiversidade [9], o consumo em excesso de carne, principalmente vermelha e processada, aumenta o risco de câncer de cólon e reto [3] e $50 \mathrm{~g}$ de carne processada pode aumentar o risco de doenças cardiovasculares em $42 \%$ e de diabetes em $19 \%{ }^{[26]}$.

Assim, a redução do consumo de carne pela população poderia beneficiar tanto o meio ambiente, diminuindo a quantidade de gases de efeito estufa para o ambiente, quanto à saúde, diminuindo o risco para as doenças crônicas que mais provocam mortes no mundo.

\section{CONCLUSÃO}

Observou-se um alto consumo de carne bovina pela população estudada, assim como alta emissão de gases de efeito estufa na sua produção. A redução de consumo de carne bovina poderia restringir pela metade os gases emitidos e diminuir o risco para doenças crônicas.

\section{AGRADECIMENTOS}

Ao Conselho Nacional de Desenvolvimento Científico e Tecnológico (CNPq no 503128/2010-4) e Fundação de Amparo à Pesquisa do Estado de São Paulo (FAPESP no 2009/15831-0) pelo financiamento.

\section{REFERÊNCIAS}

[1] Ministério da Saúde. Secretaria de Atenção à Saúde. Coordenação-Geral da Política de Alimentação e Nutrição. Guia alimentar para a população brasileira: promovendo a alimentação saudável. Brasília: Ministério da Saúde; 2006.

[2] Núcleo de Estudos e Pesquisas em Alimentação, Universidade Estadual de Campinas. Tabela brasileira de composição de alimentos. 4ạ ed. Campinas: Núcleo de Estudos e pesquisas em Alimentação, Universidade Estadual de Campinas; 2011.

[3] World Cancer Research Fund, American Institute for Cancer Research, Food, Nutrition, Physical Activity, and the Prevention of Cancer: a Global Perspective, Washington DC: AICR; 2007.

[4] McAfee AJ, McSorley EM, Cuskelly GJ, Moss BW, Wallace JMW, Bonham MP, et al. Red meat consumption: An overview of the risks and benefits. Meat Scienc. 2010;84:1-13.

[5] Daniel CR, Cross AJ, Koebnick C, Sinha R. Trends in meat consumption in the USA. Public Health Nutr. 2011;14(4):575-83.

[6] Carvalho AM, César CLG, Fisberg RM, Marchioni DML. Excessive meat consumption in Brazil: diet quality and environmental impacts. Public Health Nutr. In press.

[7] Horrigan L, Lawrende RS, Walker P. How sustainable agriculture can address environmental and human health harms of industrial agriculture. Environ Health Perspect. 2002;110:445-456.

[8] Steinfeld H, Gerber P, Wassenaar T, CastelV, Rosales M, Haan C. Livestock's long shadow: environmental issues and options. Food and Agriculture Organization of the United Nations: Roma; 2006.

[9] McMichael A, Powles JW, Butler CD, Uauy R. Food, livestock production, energy, climate change, and health. Lancet. 2007;370:1253-1263. 
[10] Garnett T. Cooking up a storm. Food, greenhouse gas emissions and our changing climate; Food Climate Research Network, Centre for Environmental Strategy, University of Surrey, 2008 [acesso em 05 junho 2012]. Disponível em: http://www.fcrn.org.uk/sites/default/files/CuaS web.pdf

[11] Cedeberg C, Persson UM, Neovius K, Molander S, Clift R. Including carbon emissions from deforestation in the carbon footprint of Brazilian beef. Environ Sci Technol. 2011;45:1773-1779.

[12] Castro MA, Barros RR, Bueno MB, César CLG, Fisberg RM. Trans fatty acids intake among the population of the city of São Paulo, Brazil. Rev Saude Pública. 2009;43:991-97.

[13] Fisberg RM, Marchioni DML. Manual para estudos populacionais de alimentação, nutrição e saúde: a experiência do inquérito de saúde em São Paulo (ISA). Grupo de Avaliação de Consumo Alimentar (GAC); 2012.

[14] Raper N, Perloff B, Ingwersen L, Steinfeldt L, Anand J. An overview of USDA's Dietary Intake Data System. Journal of Food Composition and Analysis. 2004;17:545-555.

[15] Dwyer J, Picciano MF, Raiten DJ. Future directions for the integrated CSFII, NHANES: What We Eat in America, NHANES. J Nutr. 2003;133:576S-81S.

[16] Beaton GH. Approaches to analysis of dietary data: relationship between planned analyses and choice of methodology. Am J Clin Nutr. 1994;59:253S-61S.

[17] Haubrock J, Nöthlings U, Volatier JL, Dekkers A, Ocke $\mathrm{M}$, Harttig U, et al. Estimating usual food intake distributions by using the multiple source method in the EPIC-Potsdam Calibration Study. J Nutr. 2011;1:9-920.

[18] Harttig U, Haubrock J, Knüppel S, Boeing $H$, EFCOVAL Consortium. The MSM program: web-based statistics package for estimating usual dietary intake using the Multiple Source Method. Eur J Clin Nutr. 2011;65:S87-S91.

[19] Pinheiro ABV, Lacerda EM de A, Benzecry EH, Gomes MC da S, Costa VM da. Tabela para Avaliação de Consumo Alimentar em Medidas Caseiras. 4a ed. São Paulo: Ed. Atheneu; 2000.

[20] Fisberg RM, Villar BS. Manual de receitas e Medidas caseiras para Cálculo de Inquéritos Alimentares: manual elaborado para auxiliar o processamento de inquéritos alimentares. São Paulo: Signus; 2002.

[21] NDSR - Nutrition Data System for Research. [computer program]. Version 2005. Minneapolis: University of de Minnesota; 2005.

[22] IBGE - Instituto Brasileiro de Geografia e Estatística. Indicadores IBGE: Pesquisa de orçamentos familiares 20082009: despesas, rendimentos e condições de vida. Rio de Janeiro: IBGE; 2010.

[23] Stata Corp. Stata statistical software: release 10. TX: Stata Corp LP; 2007.

[24] Carisson-Kanyama A, González AD. Potencial contributions of food consumption patterns to climate change. Am J Clin Nutr. 2009;89:1704S-9S.

[25] McMichael A, Powles JW, Butler CD, Uauy R. Food, livestock production, energy, climate change, anh health. Lancet. 2007;370:1253-1263.

[26] Micha R, Wallace SK, Mozaffarian D. Red and Processed Meat Consumption and Risk of Incident Coronary Heart Disease, Stroke, and Diabetes Mellitus: A Systematic Review and Meta-Analysis. Circulation. 2010;121:2271-83. 